\title{
Some Implications of a Scale Invariant Model of Statistical Mechanics to Boltzmann versus Shannon Entropy in Thermodynamics and Information Theory
}

\author{
SIAVASH H. SOHRAB \\ Robert McCormick School of Engineering and Applied Science \\ Department of Mechanical Engineering \\ Northwestern University, Evanston, Illinois 60208 \\ UNITED STATES OF AMERICA
}

\begin{abstract}
A scale invariant model of statistical mechanics is applied for a comparative study of Boltzmann's entropy in thermodynamics versus Shannon's entropy in information theory. The implications of the model to the objective versus subjective aspects of entropy as well as Nernst-Planck statement of the third law of thermodynamics are also discussed.
\end{abstract}

Key-Words: - Boltzmann and Shannon entropy; Information theory; Entropy; Third law of thermodynamics. Received: November 12, 2020. Revised: March 9, 2021. Accepted: April 4, 2021. Published: April 27, 2021.

\section{Introduction}

Stochastic quantum fields [1-17] and classical hydrodynamic fields [18-29] viewed as ensembles of weakly coupled oscillators resulted in the introduction of a scale-invariant model of statistical mechanics [30] and its application to the fields of thermodynamics [31], fluid mechanics [32-33], statistical mechanics [34], and quantum mechanics [35]. In the present study, some implications of the model to the physical foundation of classical and statistical thermodynamics and Boltzmann thermodynamic entropy versus Shannon information entropy as well as Nernst-Planck statement of the third law of thermodynamics are examined.

\section{A Scale-Invariant Model of Statistical Mechanics}

The scale-invariant model of statistical mechanics for equilibrium galacto-, planetary-, hydro-system-, fluid-element-, eddy-, cluster-, molecular-, atomic-, subatomic-, kromo-, and tachyon-dynamics corresponding to the scale $\beta=\mathrm{g}, \mathrm{p}, \mathrm{h}, \mathrm{f}, \mathrm{e}, \mathrm{c}, \mathrm{m}, \mathrm{a}, \mathrm{s}$, $\mathrm{k}$, and $\mathrm{t}$ is schematically shown in Fig. 1 [31]. Each statistical field is identified as the "system" and is composed of an ensemble of "elements" described by a distribution function $\mathrm{f}_{\beta}\left(\mathbf{u}_{\beta}\right)=\mathrm{f}_{\beta}\left(\mathbf{r}_{\beta}, \mathbf{u}_{\beta}, \mathrm{t}_{\beta}\right) \mathrm{d} \mathbf{r}_{\beta} \mathrm{d} \mathbf{u}_{\beta}$. Each element is composed of an ensemble of small particles called the "atoms" of the field and are viewed as point-mass. The most probable element (system) velocity of the smaller scale $(j)$ becomes the velocity of the atom (element) of the larger scale $(j+1)$.

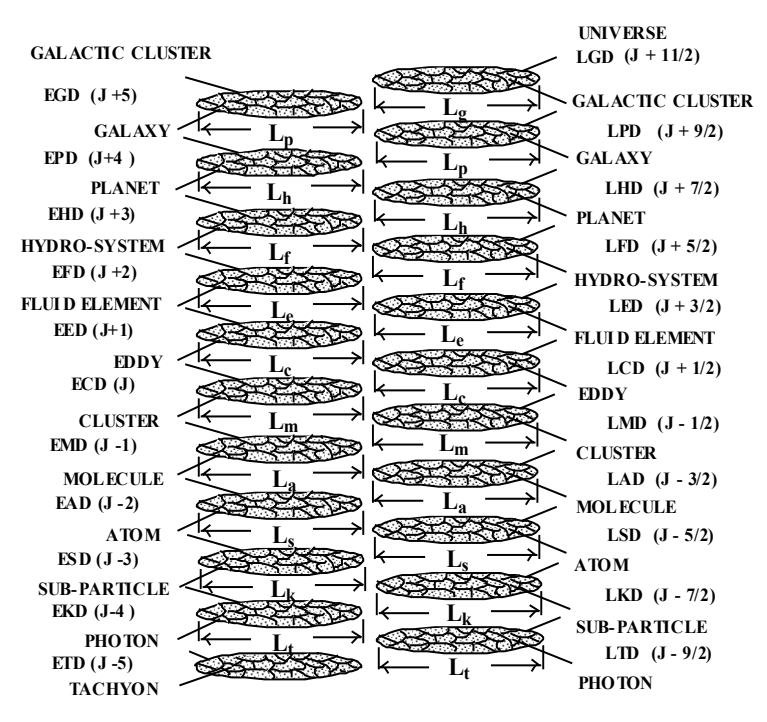

Fig. 1 A scale invariant view of statistical mechanics from cosmic to tachyon scales.

Following the classical methods [36-41] the invariant definition of density $\rho_{\beta}$, and velocity of element $\mathbf{v}_{\beta}$, atom $\mathbf{u}_{\beta}$, and system $\mathbf{w}_{\beta}$ at the scale $\beta$ are $[35,42]$

$$
\begin{array}{lll}
\rho_{\beta}=\mathrm{n}_{\beta} \mathrm{m}_{\beta}=\mathrm{m}_{\beta} \int \mathrm{f}_{\beta} \mathrm{du} u_{\beta} & , & \mathbf{u}_{\beta}=\mathbf{v}_{\mathrm{mp} \beta-1} \\
\mathbf{v}_{\beta}=\rho_{\beta}^{-1} \mathrm{~m}_{\beta} \int \mathbf{u}_{\beta} \mathrm{f}_{\beta} \mathrm{du}_{\beta} & , & \mathbf{w}_{\beta}=\mathbf{v}_{\mathrm{mp} \beta+1}
\end{array}
$$

Similarly, the invariant definition of the peculiar and diffusion velocities are introduced as 
$\mathbf{V}_{\beta}^{\prime}=\mathbf{u}_{\beta}-\mathbf{v}_{\beta} \quad, \quad \mathbf{V}_{\beta}=\mathbf{v}_{\beta}-\mathbf{w}_{\beta}$

such that

$\mathbf{V}_{\beta}=\mathbf{V}_{\beta+1}^{\prime}$

When the model is applied to social structures one arrives at the cascade shown in Fig. 2.

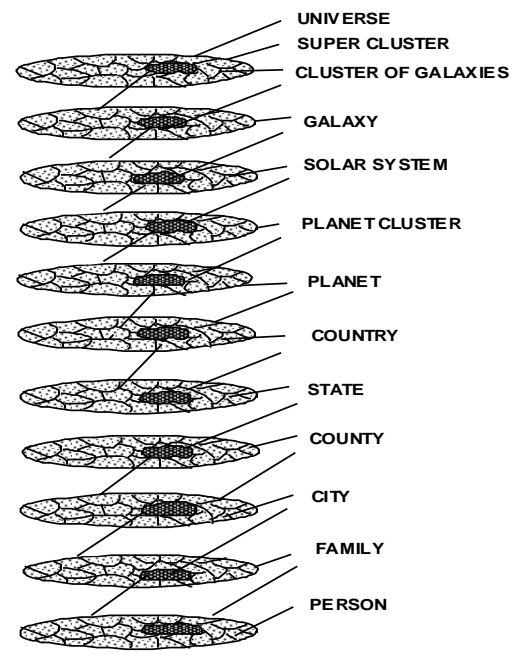

Fig. 2 Hierarchy of social structures from cosmic to individual or atomic scales.

Interestingly, as signs for capacity of number of people in elevators in Athens show, in Greek a person is referred to as "atom" that is the smallest unit of social structure shown in Fig. 2. The correspondence between statistical fields in Figs. 1-2 clearly show the physical and objective basis of information and its communication between "atoms" or individuals be it in the form of particle exchange or exchange of more complex symbols such as words or numbers. In this sense, the information theory like all other branches of science must of course be a subset of the theory of everything TOE (Fig. 1).

\section{Stochastic Natures of Planck and Boltzmann Constants and De Pretto Number 8338}

Because at thermodynamic equilibrium the mean velocity of each particle, Heisenberg-Kramers virtual oscillator [43], vanishes $\left\langle\mathbf{u}_{\beta}\right\rangle=0$ the translational kinetic energy of particle oscillating in two directions $\left(\mathrm{x}^{+}, \mathrm{x}^{-}\right)$is expressed as

$$
\begin{aligned}
\varepsilon_{\beta}= & \mathrm{m}_{\beta}\left\langle\mathrm{u}_{\beta \mathrm{x}+}^{2}\right\rangle / 2+\mathrm{m}_{\beta}\left\langle\mathrm{u}_{\beta \mathrm{x}-}^{2}\right\rangle / 2 \\
& =\mathrm{m}_{\beta}\left\langle\mathrm{u}_{\beta \mathrm{x}+}^{2}\right\rangle=\overline{\mathrm{p}}_{\beta}\left\langle\lambda_{\beta}^{2}\right\rangle^{1 / 2}\left\langle v_{\beta}^{2}\right\rangle^{1 / 2}
\end{aligned}
$$

where $\quad \bar{p}_{\beta}=m_{\beta}\left\langle u_{\beta x+}^{2}\right\rangle^{1 / 2}$ is the root-mean-square momentum of particle and $\left\langle\mathrm{u}_{\beta \mathrm{x}+}^{2}\right\rangle=\left\langle\mathrm{u}_{\beta \mathrm{x}}{ }^{2}\right\rangle$ by Boltzmann equipartition principle. At any scale $\beta$, the result in Eq. (5) can be expressed in terms of either frequency or wavelength

$$
\begin{gathered}
\varepsilon_{\beta}=\mathrm{m}_{\beta}\left\langle\mathrm{u}_{\beta}^{2}\right\rangle=\overline{\mathrm{p}}_{\beta}\left\langle\lambda_{\beta}^{2}\right\rangle^{1 / 2}\left\langle v_{\beta}^{2}\right\rangle^{1 / 2}=\mathrm{h}_{\beta} v_{\beta} \\
\varepsilon_{\beta}=\mathrm{m}_{\beta}\left\langle\mathrm{u}_{\beta}^{2}\right\rangle=\overline{\mathrm{p}}_{\beta}\left\langle v_{\beta}^{2}\right\rangle^{1 / 2}\left\langle\lambda_{\beta}^{2}\right\rangle^{1 / 2}=\mathrm{k}_{\beta} \lambda_{\beta}
\end{gathered}
$$

when the definition of stochastic Planck and Boltzmann factors are introduced as [33]

$$
\begin{aligned}
& \mathrm{h}_{\beta}=\overline{\mathrm{p}}_{\beta}\left\langle\lambda_{\beta}^{2}\right\rangle^{1 / 2} \\
& \mathrm{k}_{\beta}=\overline{\mathrm{p}}_{\beta}\left\langle v_{\beta}^{2}\right\rangle^{1 / 2}
\end{aligned}
$$

At the important scale of EKD (Fig. 1) corresponding to Casimir [44] vacuum composed of photon gas, the universal constants of Planck [45, 46] and Boltzmann [31] are identified from equations (6)-(7) as

$$
\begin{aligned}
& \mathrm{h}=\mathrm{h}_{\mathrm{k}}=\mathrm{m}_{\mathrm{k}} \mathrm{c}\left\langle\lambda_{\mathrm{k}}^{2}\right\rangle^{1 / 2}=6.626 \times 10^{-34} \mathrm{~J}-\mathrm{s} \\
& \mathrm{k}=\mathrm{k}_{\mathrm{k}}=\mathrm{m}_{\mathrm{k}} \mathrm{c}\left\langle v_{\mathrm{k}}^{2}\right\rangle^{1 / 2}=1.381 \times 10^{-23} \mathrm{~J} / \mathrm{K}
\end{aligned}
$$

Next, following de Broglie hypothesis for the wavelength of matter waves [2]

$\lambda_{\beta}=\mathrm{h} / \overline{\mathrm{p}}_{\beta}$

the frequency of matter waves is defined as [31]

$v_{\beta}=\mathrm{k} / \overline{\mathrm{p}}_{\beta}$

When matter and radiation are in the state of thermodynamic equilibrium equations (9a) and (9b) can be expressed as

$\mathrm{h}_{\beta}=\mathrm{h}_{\mathrm{k}}=\mathrm{h} \quad, \quad \mathrm{k}_{\beta}=\mathrm{k}_{\mathrm{k}}=\mathrm{k}$

The definitions in equations (8a) and (8b) result in the gravitational mass of photon [31]

$$
\mathrm{m}_{\mathrm{k}}=\left(\mathrm{hk} / \mathrm{c}^{3}\right)^{1 / 2}=1.84278 \times 10^{-41} \mathrm{~kg}
$$

that is much larger than the reported [47] value of $4 \times 10^{-51} \mathrm{~kg}$. The finite gravitational mass of photons was anticipated by Newton [48] and is in accordance with Einstein-de Broglie theory of light [49-53]. Avogardo-Loschmidt number was predicted as [31]

$$
\mathrm{N}^{\mathrm{o}}=1 /\left(\mathrm{m}_{\mathrm{k}} \mathrm{c}^{2}\right)=6.0376 \times 10^{23}
$$

leading to the modified value of the universal gas constant 


$$
\mathrm{R}^{\mathrm{o}}=\mathrm{N}^{\mathrm{o}} \mathrm{k}=8.338 \mathrm{~kJ} /(\mathrm{kmol}-\mathrm{K})
$$

Also, by Eq. (13) the atomic mass unit becomes

$$
\begin{aligned}
\mathrm{amu} & =\mathrm{m}_{\mathrm{k}} \mathrm{c}^{2} \\
& =(\mathrm{hkc})^{1 / 2}=1.6563 \times 10^{-27} \mathrm{~kg} / \mathrm{kmol}
\end{aligned}
$$

Since all baryonic matter is known to be composed of atoms, equations (12) and (15) suggest that all matter in the universe is composed of light [54]. From Eqs. (8a)-(8b) the wavelength and frequency of photon in vacuum $\left\langle\lambda_{\mathrm{k}}^{2}\right\rangle^{1 / 2}\left\langle v_{\mathrm{k}}^{2}\right\rangle^{1 / 2}=\mathrm{c}$ are

$$
\begin{gathered}
\lambda_{\mathrm{k}}=\left\langle\lambda_{\mathrm{k}}^{2}\right\rangle^{1 / 2}=1 / \mathrm{R}^{\mathrm{o}}=0.119935 \mathrm{~m}, \\
v_{\mathrm{k}}=\left\langle v_{\mathrm{k}}^{2}\right\rangle^{1 / 2}=2.49969 \times 10^{9} \mathrm{~Hz}
\end{gathered}
$$

In a recent study [35] a modified definition of thermodynamic temperature $\mathrm{T}^{\prime}=2 \mathrm{~T}$ was introduced that resulted in the modified value of Joule-Mayer mechanical equivalent of heat $\mathrm{J}$ [35]

$$
\mathrm{J}=2 \mathrm{~J}_{\mathrm{c}}=2 \times 4.169=8338 \quad \text { Joule } / \mathrm{kcal}
$$

where the classical value $J_{c}=4.169 \simeq 4.17[\mathrm{~kJ} / \mathrm{kcal}]$ is the average of two values $J_{c}=(4.15,4.19)$ reported by Pauli [55]. The number in Eq. (17) is thus identified as the universal gas constant in Eq. (14) when expressed in appropriate MKS system of units

$$
\mathrm{R}^{\mathrm{o}}=\mathrm{kN}^{\mathrm{o}}=\mathrm{J}=8338 \quad \text { Joule } /(\mathrm{kmol}-\mathrm{K})
$$

The modified value of the universal gas constant in Eq. (14) was recently identified [56] as De Pretto number 8338 that appeared in the mass-energy equivalence equation of De Pretto [57]

$$
\mathrm{E}=\mathrm{mc}^{2} \text { Joule }=\mathrm{mc}^{2} / 8338 \mathrm{kcal}
$$

Unfortunately, the name of Olinto De Pretto in the history of evolution of mass energy equivalence is little known. Ironically, Einstein's best friend Michele Besso was a relative and close friend of Olinto De Pretto's brother Augusto De Pretto. The relativistic form of equation (19) was first introduced in 1900 by Poincaré [58]

$$
\mathrm{E}=\mathrm{m}_{\mathrm{r}} \mathrm{c}^{2}
$$

where $\mathrm{m}_{\mathrm{r}}=\mathrm{m}_{\mathrm{o}} / \sqrt{1-\mathrm{v}^{2} / \mathrm{c}^{2}}$. Since the expression (19) is the only equation in the paper by De Pretto [57], the exact method by which he arrived at the number 8338 is not known even though one possible method was recently suggested [56]. The important contributions by Hasenöhrl [59] and Einstein [60] as well as the equivalence principle, equivalence of the rest or gravitational mass and the inertial mass were discussed in a recent study [54].

According to Eq. (15) mass is simply associated with numbers in harmony with the perceptions of Sommerfeld [61]

"Our spectral series, dominated as they are by integral quantum numbers, correspond, in a sense, to the ancient triad of the lyre, from which the Pythagoreans 2500 years ago inferred the harmony of the natural phenomena; and our quanta remind us of the role which the Pythagorean doctrine seems to have ascribed to the integers, not merely as attributes, but as the real essence of physical phenomena."

as well as the ideas of Weyl [62].

\section{Objective versus Subjective Nature of Thermodynamic Entropy}

Possible subjective versus objective nature of entropy has been subject of much debate ever since 1948 when Shannon [63-64] used the name entropy in his information theory. The objective nature of thermodynamic entropy is evident form its classical definition first introduced by Clausius

$\mathrm{d} S=\mathrm{d} Q_{\text {rev }} / \mathrm{T}$

In other words, direct connection between entropy $S$ and heat $Q$ clearly establishes the objective nature of the former.

The misunderstandings concerning possible subjective nature of entropy could be due to its statistical definition first introduced by Boltzmann

$S=\mathrm{k} \ln \mathrm{W} \quad, \quad S_{\mathrm{j}}=\mathrm{k} \ln \mathrm{W}_{\mathrm{j}}$

The total number of complexions for independent energy levels introduced by Boltzmann [65-67] and Planck [45] is

$$
\mathrm{W}=\prod_{\mathrm{j}} \mathrm{W}_{\mathrm{j}}
$$

The number of complexions for distributing $\mathrm{N}_{\mathrm{j}}$ indistinguishable particles among $\mathrm{g}_{\mathrm{j}}$ distinguishable clusters or "quantum states" for level (element) $j$ is

$\mathrm{W}_{\mathrm{j}}=\frac{\left(\mathrm{N}_{\mathrm{j}}+\mathrm{g}_{\mathrm{j}}-1\right) !}{\mathrm{N}_{\mathrm{j}} !\left(\mathrm{g}_{\mathrm{j}}-1\right) !}$

According to the present model of statistical mechanics $g_{j}$ denotes number of distinguishable clusters (elements) in a particular energy level $\mathrm{j}$ of the system [35]. However, the smallest cluster contains only a single particle and is therefore considered to be full since no other particle can be added to this smallest cluster. Because an empty 
cluster has no physical significance, the total number of available clusters or quantum states will be $\left(\mathrm{g}_{\mathrm{j}}-1\right)$. Therefore, Planck-Boltzmann formula (24) is the exact probability of distribution of $\mathrm{N}_{\mathrm{j}}$ indistinguishable oscillators amongst $\left(g_{\mathrm{j}}-1\right)$ distinguishable available clusters. The invariant model of statistical mechanics (Fig. 1) provides new perspectives on the probabilistic nature of Eq. (24) and the problem of distinguishability discussed by Darrigol [68].

Under the realistic assumptions

$\mathrm{g}_{\mathrm{j}} \gg \mathrm{N}_{\mathrm{j}} \quad, \quad \mathrm{N}_{\mathrm{j}} \gg 1$

it is known that the number of complexions for Bose-Einstein statistics in Eq. (24) simplifies such that all three types namely Corrected MaxwellBoltzmann, Bose-Einstein, and Fermi-Dirac statistics will have [69]

$$
\mathrm{W}_{\mathrm{j}}=\mathrm{N}_{\mathrm{j}}^{\mathrm{N}_{\mathrm{j}}} / \mathrm{N}_{\mathrm{j}} \text { ! } \quad, \quad \mathrm{W}=\mathrm{N}^{\mathrm{N}} / \mathrm{N} \text { ! }
$$

According to the classical methods the maximization of $\mathrm{W}$ in Eq. (23) will result in the Boltzmann distribution [42]

$$
N_{j}=g_{j} e^{-\beta\left(\varepsilon_{j}-\alpha \hat{\mu}_{j}\right)}=g_{j} e^{-\left(\varepsilon_{j}-\alpha \hat{\mu}_{j}\right) / k T}
$$

The coefficient $\alpha$ multiplying chemical potential $\hat{\mu}_{\mathrm{j}}$ was shown to be unity $\alpha=1$ [42] and the partition function for normalization is defined as

$$
\mathrm{Z}=\sum \mathrm{g}_{\mathrm{j}} \mathrm{e}^{-\beta\left(\varepsilon_{\mathrm{j}}-\alpha \hat{\mu}_{\mathrm{j}}\right)}
$$

According to the information theory of Shannon [67-68] the amount of information $\mathrm{H}$ also referred to as "entropy" is expressed by Shannon formula

$$
\mathrm{H}_{\max }=-\mathrm{K} \sum_{\mathrm{j}} p_{\mathrm{j}} \ln p_{\mathrm{j}}
$$

where $\mathrm{K}$ is a positive constant. The above definition is based on conditional probability for occurrence of event $\mathrm{j}$ given a message $\mathrm{A}$ [69]

$\hat{i}=\mathrm{K} \ln \left[p_{\mathrm{j}}(\mathrm{j} / \mathrm{A}) / p_{\mathrm{j}}\right]$

Only with maximum value of the conditional probability

$$
p_{\mathrm{j}}(\mathrm{j} / \mathrm{A})=1
$$

equation (31) reduces to

$$
\hat{i}_{\max }=-\mathrm{K} \ln p_{\mathrm{j}}
$$

thus leading to the mean value of information entropy [69]

$$
\mathrm{H}_{\text {max }}=\sum_{\mathrm{j}} \hat{i}_{\max } p_{\mathrm{j}}=-\mathrm{K} \sum_{\mathrm{j}} p_{\mathrm{j}} \ln p_{\mathrm{j}}
$$

The thermodynamic "entropy" on the other hand is often derived on the basis of number of complexions for distribution of particles amongst various quantum states expressed as

$\mathrm{W}=\mathrm{N} ! / \prod_{\mathrm{j}} \mathrm{N}_{\mathrm{j}}$ !

The $\mathrm{W}$ in Eq. (35) along with the definition of probability

$p_{\mathrm{j}}=\mathrm{N}_{\mathrm{j}} / \mathrm{N}$

when substituted in Boltzmann equation (22) lead to "entropy" [69]

$S=\mathrm{Nk} \ln \mathrm{W}=-\mathrm{Nk} \sum_{\mathrm{j}} p_{\mathrm{j}} \ln p_{\mathrm{j}}$

Clearly, Eq. (37) does not represent the true thermodynamic entropy of Boltzmann and the discrepancy arises from the fact that $\mathrm{W}$ in Eq. (35) does not correspond to that in Eq. (23).

It is desirable to avoid the above mentioned discrepancy between Eq. (37) and the true thermodynamic entropy of Boltzmann. In other words, one wishes to establish the exact relationship between Shannon's entropy of information in Eq. (30) and the true thermodynamic entropy that represents the degree of randomness of energy distributions of particles amongst particle clusters, de Broglie wave packets, in statistical fields as measured by the number of complexions according to Boltzmann equation (23). Therefore, rather than the conventional definition in Eq. (36) the thermodynamic probability $p_{\mathrm{j}}$ is defined as

$p_{\mathrm{j}}=\mathrm{W}_{\mathrm{j}} / \mathrm{W}$

Next, since Boltzmann entropy represents a measure of uncertainty or randomness from Eq. (22) and in harmony with ideas of Brillouin [70] the difference between maximum entropy and entropy of state $\mathrm{j}$ is defined as a measure of information that by Eq. (38) gives

$$
\mathrm{I}_{\mathrm{jmax}}=S_{\max }-\mathrm{S}_{\mathrm{j}}=-\mathrm{k} \ln p_{\mathrm{j}}
$$

Since reduction of uncertainty from its maximum value to that of state $\mathrm{j}$ is equivalent to an increase of certainty it constitutes information as schematically shown in Fig. 3. 


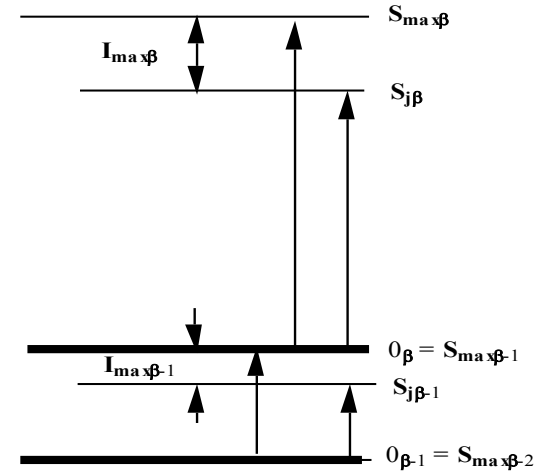

Fig. 3 Scale-invariant definition of thermodynamic information defined as reduction of absolute uncertainty or entropy $I_{\max }=S_{\max \beta}-S_{\mathrm{j} \beta}$.

Hence, parallel to Eq. (34) the mean value of thermodynamic information becomes

$$
\mathrm{I}_{\max }=\sum_{\mathrm{j}} \mathrm{I}_{\mathrm{j} \max } p_{\mathrm{j}}=-\mathrm{k} \sum_{\mathrm{j}} p_{\mathrm{j}} \ln p_{\mathrm{j}}
$$

The equations (30) and (40) now only differ by the universal Boltzmann constant k.

The occurrence of the universal constant $\mathrm{k}$ has fundamental significance. According to equations (7)-(8) Planck and Boltzmann constants are two fundamental constants of nature that relate to spatial and temporal aspect of Casimir [44] vacuum fluctuations. Because all conceivable information must be transmitted by some physical entity, such as electron, photon, or neutrino, etc. in space-time, it is reasonable to expect that both constants $(\mathrm{h}, \mathrm{k})$ will play a central role is transmission of information. Therefore, it is not advisable to modify thermodynamic entropy in Eq. (40) to achieve correspondence with Eq. (30). Instead one can simply define dimensionless Boltzmann thermodynamic information entropy as

$$
\tilde{\mathrm{H}}_{\max }=\mathrm{I}_{\max } / \mathrm{k}=-\sum_{\mathrm{j}} p_{\mathrm{j}} \ln p_{\mathrm{j}}
$$

in exact correspondence with dimensionless Shannon information entropy in Eq. (30).

The classical definition of entropy by Boltzmann is based on the number of complexions according to equations (22)-(24). However, it is possible to introduce a modified definition of thermodynamic entropy as

$$
S_{\mathrm{j}}=-\mathrm{k} \ln p_{\mathrm{j}}
$$

that is exactly equivalent to thermodynamic entropy of Boltzmann described in an earlier study [42]. To arrive at the modified definition in Eq. (42) one first introduces the thermodynamic probability as

$p_{\mathrm{j}}=\mathrm{N}_{\mathrm{j}} / \mathrm{N}$

that will be directly related to thermodynamic equilibrium distribution function of Boltzmann in Eq. (28) normalized as

$\mathrm{N}_{\mathrm{j}}=\mathrm{Ne}^{-\left(\mathrm{E}_{\mathrm{j}}-\mathrm{E}\right) / \mathrm{kT}}=\mathrm{Ne}^{-H_{\mathrm{j}} / \mathrm{kT}}$

The simplification in Eq. (44) is due to the vanishing of chemical potential $\hat{\mu}_{\mathrm{j}}=0$ of ideal gas at equilibrium by to the equilibrium conditions $G=\mathrm{d} G$ $=0$ [42]. Next, one identifies the energy $\left(E_{j}-E\right)$ in Eq. (44) as the transition energy or enthalpy $H_{\mathrm{j}}$ of energy level $\mathrm{j}$ that for an ideal gas is given by

$$
H_{\mathrm{j}}=U_{\mathrm{j}}+\mathrm{p}_{\mathrm{j}} V=3 \mathrm{~N}_{\mathrm{j}} \mathrm{kT}+\mathrm{N}_{\mathrm{j}} \mathrm{kT}=4 \mathrm{~N}_{\mathrm{j}} \mathrm{kT}
$$

Because Boltzmann distribution in Eq. (28) is obtained from maximization of $\mathrm{W}_{\mathrm{j}}$ one can relate entropy to the probability $p_{\mathrm{j}}$ based on Boltzmann distribution obtained from equations (43) - (45) as

$$
p_{\mathrm{j}}=\mathrm{N}_{\mathrm{j}} / \mathrm{N}=\mathrm{e}^{-H_{\mathrm{j}} / \mathrm{kT}}=\mathrm{e}^{-4 \mathrm{~N}_{\mathrm{j}}}
$$

Finally, by substitution from Eq. (46) into Eq. (42) one obtains

$S_{\mathrm{j}}=4 \mathrm{~N}_{\mathrm{j}} \mathrm{k}$

in exact agreement with the result obtained based the number of complexions [42]. Therefore, the reason for the validity of equivalence of equations (22) and (42) is that equations (43) and (46) are connected to thermodynamic equilibrium through Boltzmann distribution in Eq. (44) that in turn was derived on the basis of maximization of $\mathrm{W}_{\mathrm{j}}$ [42].

The probability function in Eq. (46) and its origination from the Boltzmann distribution function in Eq. (44) helps to clarify the nature of the connection between thermodynamic entropy of Boltzmann and the information entropy of Shannon. To better reveal this connection, one first notes that the concept of entropy according to Boltzmann's statistical definition in Eq. (22) concerns maximization of randomness by maximization of number of complexions W. For example, when applied to photon gas representing equilibrium black-body radiation maximization of $\mathrm{W}$ will correspond to having a spectrum of photon cluster sizes, de Broglie molecules of light, with energy distribution given by Planck energy spectrum [35, 
42]. It is noted that the entropy of equilibrium photon gas given by Eq. (47) involves number $\mathrm{N}_{\mathrm{j}}$ of Planck or Heisenberg-Kramers virtual oscillators, as well as the universal constant of Boltzmann such that the total energy of the energy-level $j$ may be expressed as [42]

$$
\mathrm{T}_{\mathrm{j}} S_{\mathrm{j}}=4 \mathrm{~N}_{\mathrm{j}} \mathrm{kT}_{\mathrm{j}}=4 \mathrm{~N}_{\mathrm{j}} \bar{\varepsilon}_{\mathrm{tj}}=4 \mathrm{~N}_{\mathrm{j}} \frac{\hat{\mathrm{u}}_{\mathrm{j}}}{3}=\frac{4}{3} U_{\mathrm{j}}
$$

In Eq. (48) the mean energy of oscillator involving the product $\bar{\varepsilon}_{\mathrm{tj}}=\mathrm{kT}_{\mathrm{j}}$ is divided into two parts the first part $4 \mathrm{~N}_{\mathrm{j}} \mathrm{k}$ is the definition of entropy by Eq. (47) and the second part $T_{j}$ relates to the de Broglie wavelength of oscillator by Eq. (6b) as

$$
\bar{\varepsilon}_{\mathrm{t} \beta}=\overline{\mathrm{p}}_{\beta}\left\langle\mathrm{v}_{\beta}^{2}\right\rangle^{1 / 2}\left\langle\lambda_{\beta}^{2}\right\rangle^{1 / 2}=\mathrm{k}_{\beta} \lambda_{\beta}=\mathrm{kT}_{\beta}
$$

As a result the definitions of $(\mathrm{k}, \mathrm{T})$ lead to conjugate roles of thermodynamic properties $(S, \mathrm{~T})$ such that their product gives the oscillator energy in Eq. (48).

Now that the role of constant $\mathrm{k}$ in Boltzmann thermodynamic entropy has been identified, possible role of the universal constant $\mathrm{k}$ in Shannon information entropy will be examined. First, it is noted that all communications and exchange of information must involve some kind of physical entity such as a particle like molecule, atom, electron, photon, neutrino, ... involving moleculardynamics, ... electro-dynamics, chromo-dynamics, ... statistical fields. Such fundamental approach to communication theory can also address the problem of conveying information by ensembles of different types of symbols such as alphabets, A, B, C, ... and alphabet clusters containing $\mathrm{N}_{\mathrm{A}}, \mathrm{N}_{\mathrm{B}}, \mathrm{N}_{\mathrm{C}}, \ldots$ alphabets such as encountered in the information theory of Shannon [67, 68]. Hence, parallel to system of ideal gas, one seeks a spectrum of "alphabet cluster" or "word" sizes that will maximize the entropy i.e. total randomness of the system such that the number of complexions expressed by corresponding Boltzmann-Planck Eq. (24) is maximized. Under "equilibrium" distribution alphabet-cluster sizes will correspond to maximum randomness and hence entropy and thus minimum information in accordance with Fig. 3. It is now clear that in order to insure an exact correspondence between Boltzmann and Shannon entropy one must let $\mathrm{K}=\mathrm{k}$ and write Shannon formula Eq. (33) as

$$
\hat{i}_{\max }=-\mathrm{k} \ln p_{\mathrm{j}}
$$

Indeed, concerning the positive constant $\mathrm{K}$ in his formula in Eq. (33) Shannon writes [63- 64]
"The choice of the coefficient $K$ is a matter of convenience and amounts to the choice of a unit of measure"

Equation (50) leads to modified Shannon formula

$$
\mathrm{H}_{\max }=\sum_{\mathrm{j}} \hat{i}_{\max } p_{\mathrm{j}}=-\mathrm{k} \sum_{\mathrm{j}} p_{\mathrm{j}} \ln p_{\mathrm{j}}
$$

that is now in exact correspondence with Boltzmann formula in Eq. (40). The information entropy in Eq. (51) insures that the conjugate thermodynamic properties $(S, \mathrm{~T})$ are such that their product $\mathrm{T} S$ will relate to the system energy as expressed in Eq. (48). The Boltzmann constant $\mathrm{k}=\mathrm{K}$ in Eq. (51) does indeed relate to the choice of the "measure" as was anticipated by Shannon [63-64]. This is because according to the present theory thermodynamic temperature is identified as a length scale $\mathrm{T}=\left\langle\lambda_{\beta}^{2}\right\rangle^{1 / 2}$ that is de Broglie wavelength according to equations (6) and (9). Therefore, in exact agreement with the perceptions of Shannon as described in the above quotation, the constant $\mathrm{K}=\mathrm{k}$ is related to the measure $\mathrm{T}=\left\langle\lambda_{\beta}^{2}\right\rangle^{1 / 2}$ since the product $\mathrm{kT}$ gives the mean particle energy according to Eq. (49). In other words, the introduction of $\mathrm{k}$ in Eq. (51) is necessary since it allows the entropy of information to be related to energy through de Broglie wavelength of the particles being used to transmit the information.

Recently, it was suggested by Ben-Naim [71] that to achieve exact correspondence between Boltzmann's entropy in thermodynamics in Eq. (40) and Shannon's entropy in information theory in Eq. (34) the dimension of thermodynamics absolute temperature be changed

"One should redefine a new absolute
temperature; denote it tentatively as $\bar{T}$ to replace
$k T$. The new temperature $\bar{T}$ would have the units
of energy and there would be no need for
Boltzmann constant. The equation for entropy
will be simply $S=\ln W,{ }^{21}$ and entropy would be
rendered dimensionless"; "This will automatically
expunge the Boltzmann constant $k_{B}$ from the
vocabulary of physics"

According to the definitions in equations (5)(10) of Section 3 Planck $\mathrm{h}$ and Boltzmann $\mathrm{k}$ constants are associated with the stochastically stationary aspects of Casimir [72] vacuum fluctuations with the dimensions

$$
\begin{array}{lc}
\varepsilon_{\beta}=\mathrm{h}\left\langle v_{\beta}^{2}\right\rangle^{1 / 2}=\mathrm{h}_{\beta} v_{\beta} & {[\mathrm{J}-\mathrm{s}] \cdot[\mathrm{Hz}]=[\mathrm{J}]} \\
\varepsilon_{\beta}=\mathrm{k}\left\langle\lambda_{\beta}^{2}\right\rangle^{1 / 2}=\mathrm{k}_{\beta} \lambda_{\beta} & {[\mathrm{J} / \mathrm{m}] .[\mathrm{m}]=[\mathrm{J}]}
\end{array}
$$


Therefore, just like Planck constant $\mathrm{h}$ what he named Boltzmann constant $\mathrm{k}$ cannot be expunged from the vocabulary of physics. Also, according to Eq. (6b) Kelvin absolute temperature is identified as a length scale associated with mean wavelength of thermal oscillations.

$\varepsilon_{\beta}=\mathrm{k}\left\langle\lambda_{\beta}^{2}\right\rangle^{1 / 2}=\mathrm{kT}$

The identification of dimension of absolute thermodynamic temperature as [meter] rather than [degree $\mathrm{K}$ ] is a step towards clarification of the exact physical nature of this intensive property.

Because temperature is a measured quantity ultimately it is the theory that must determine the significance of such a variable and its dimension according to Planck [73].

"Every measurement first acquires its meaning for physical science through the significance which a theory gives it"

Therefore, following Planck's suggestion, it is reasonable to change the dimension of absolute thermodynamic temperature $\mathrm{T}$ from [degree $\mathrm{K}$ ] to [meter]. The exact correspondence between $\mathrm{H}_{\max }$ and $I_{\max }$ could be achieved through the definition of dimensionless thermodynamic information entropy $\tilde{\mathrm{H}}_{\max }$ in Eq. (41).

Arguments have also been made that the very name entropy first coined by its discoverer Clausius should be changed [74]

"It is also time to change not only the units of entropy to make it dimensionless, but the term "entropy" altogether. Entropy, as is now recognized, does not mean "transformation, or "change" or "turn". It does mean information. Why not replace the term that means "nothing" as Cooper noted, and does not even convey the meaning it was meant to convey when selected by Clausius? Why not replace it with a simple, familiar, meaningful, and precisely defined term "information?"

It is emphasized however that contrary to the above quotation the name entropy has been chosen most appropriately by Clausius to represent precisely what it should namely "transformation". According to the result in Eq. (47) for ideal gas, entropy relates to the number of Planck [45] or Heisenberg-Kramers oscillators [43]. Therefore, the second law of thermodynamics suggests that in all natural processes there is a tendency to transform energy and hence motion from ordered motions of a few large-scale oscillators to random motions of many small-scale oscillators thus increasing the entropy of the system. In other words entropy according to its macroscopic definition by Clausius is directly related to heat

$\mathrm{d} S=\mathrm{d} Q_{\text {rev }} / \mathrm{T}$

Therefore, all types of energy dissipation induced by plastic deformation, friction, viscosity,... will lead to transformation of ordered (correlated) motions into disordered (uncorrelated) random motions that is heat thus leading to increased entropy by Eq. (54). It seems that if we follow the changes of dimensions and notations suggested by Ben-Naim [71, 74] the state of our scientific terminology may become void and hence approach what he calls "Tohu Vavohu" [71] meaning total chaos.

\section{Impact on Nernst-Planck Third Law of Thermodynamics}

According to Nernst-Planck statement of the third law of thermodynamics, entropy must approach zero as absolute thermodynamic temperature approaches zero. Another statement of the third law is [75]

\section{"It is impossible to reach absolute zero using a finite number of processes"}

or the absolute zero temperature cannot be reached with a finite number of steps. Examination of Fig. 1 clearly shows that the very definitions of space and time that is applicable across the hierarchies of embedded statistical fields become complicated [35] and demand careful investigations. Recently, it was suggested [42] that the description of the hierarchy of statistical fields shown in Fig. 1 could be expressed in terms of a logarithmic coordinates schematically shown in Fig. 4.

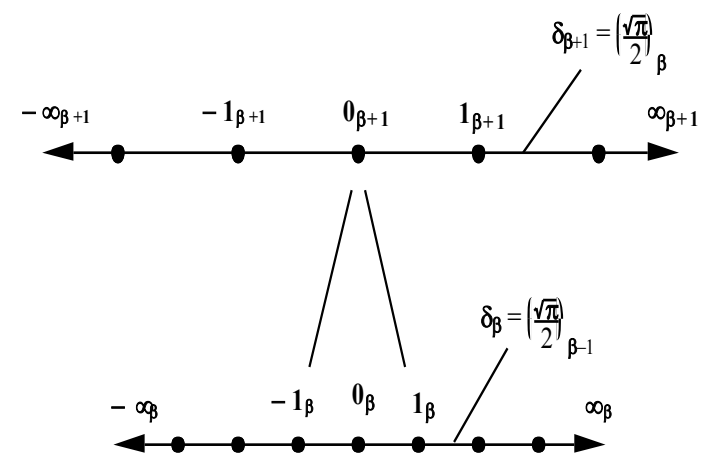

Fig. 4 Hierarchy of normalized coordinates associated with embedded statistical fields [42].

Because according to Eq. (53) thermodynamic temperature is identified as a length scale in view of Fig. 4 one arrives at a hierarchy of absolute zero temperatures [42] 


$$
\begin{array}{r}
\mathrm{T}_{\beta}=0_{\beta}=\mathrm{T}_{\beta-1}=1_{\beta-1} \\
\mathrm{~T}_{\beta-1}=0_{\beta-1}=\mathrm{T}_{\beta-2}=1_{\beta-2} \\
\ldots
\end{array}
$$

Furthermore, entropy of an ideal gas is a measure of the number of oscillators by Eq. (47). Therefore, from Nernst-Planck statement of the third law and Eq. (55) one arrives at the hierarchy of "absolute zero" temperature and corresponding "absolute zero" entropy schematically shown in Fig. 5.

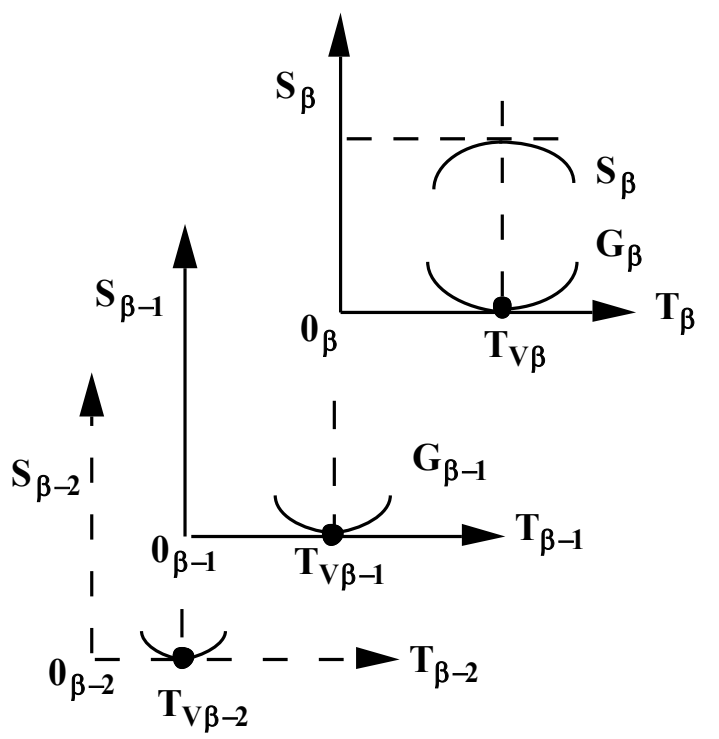

Fig. 5 Hierarchy of absolute "zeros" $T_{\beta}=0_{\beta}$ and "vacuum" $T_{\mathrm{v} \beta}$ temperatures and associated entropies approached at equilibrium corresponding to minimum $G i b b s$ free energy $G_{\beta}$ or maximum entropy $S_{\beta}[42]$.

The hierarchy shown in Fig. 5 is associated with the hierarchy of vacua at equilibrium conditions and the associated entropy and enthalpy leading to the vanishing of invariant Gibbs free energy

$$
G_{\mathrm{v} \beta}=H_{\mathrm{v} \beta}-\mathrm{T}_{\mathrm{v} \beta} S_{\mathrm{v} \beta}=0_{\beta}
$$

as discussed in Sec. 10 of an earlier study [42].

\section{Concluding Remarks}

A scale-invariant model of statistical mechanics was applied to describe the nature of the connection between Boltzmann thermodynamic entropy and Shannon information entropy. Also, the application of the model to statistical fields associated with social structures was described. It was shown that the equality of the positive constant $\mathrm{K}$ in Shannon's formula for information entropy and the Boltzmann constant k in Boltzmann formula for thermodynamic entropy insure the consistency between the energy and de Broglie wavelengths of particles being used to transmit information. Finally, the impact of the model on the Nernst-Planck statement of the third law of thermodynamics was briefly discussed.

\section{Acknowledgements:}

This research was in part supported by NASA grant No. NAG3-1863.

\section{References:}

[1] Broglie, L. de, "Interference and Corpuscular Light," Nature 118, 2969, 1926, pp. 441-442; “ Sur la Possibilité de Relier les Phénomènes d'Interférence et de Diffraction à la Théorie des Quanta de Lumière," C. R. Acad. Sci. Paris, 183, 1927, pp. 447-448; "La Structure Atomique de la Matière et du Rayonnement et la Mécanique Ondulatoire," 184, 1927, pp. 273-274; "Sur le Rôle des Ondes Continues en Mécanique Ondulatoire," 185, 1927, pp. 380-382

[2] Broglie, L. de, Non-Linear Wave Mechanics: A Causal Interpretation, Elsevier, New York, 1960

[3] Broglie, L. de, "The Reinterpretation of Wave Mechanics," Found. Phys. 1, 5, 1970, pp. 5-15.

[4] Madelung, E., "Quantentheorie in Hydrodynamischer Form," Z. Physik. 40, 1926, pp. 332-326.

[5] Schrödinger, E., "Über die Umkehrung der Naturgesetze," Sitzber Preuss Akad Wiss PhysMath Kl, 1931, p. 144-153.

[6] Fürth, R., "Über Einige Beziehungen zwischen klassischer Staristik und Quantenmechanik," $Z$. Phys. 81, 1933, pp. 143-162.

[7] Bohm, D., "A Suggested Interpretation of the Quantum Theory in Terms of "Hidden" Variables. I" Phys. Rev. 85, 2, 1952, pp.166-179.

[8] Takabayasi, T., "On the Foundation of Quantum Mechanics Associated with Classical Pictures," Prog. Theor. Phys. 8, 2, 1952, pp. 143-182.

[9] Bohm, D., and Vigier, J. P., "Model of the Causal Interpretation of Quantum Theory in Terms of a Fluid with Irregular Fluctuations," Phys. Rev. 96, 1, 1954, pp. 208-217.

[10] Nelson, E. "Derivation of the Schrödinger Equation from Newtonian Mechanics," Phys. Rev. 150, 4, 1966, pp. 1079-1085.

[11] Nelson, E., Quantum Fluctuations, Princeton University Press, Princeton, New Jersey, 1985.

[12] de la Peña, L., "New Foundation of Stochastic Theory of Quantum Mechanics," J. Math. Phys. 10, 9, 1969, pp. 1620-1630.

[13] de la Peña, L., and Cetto, A. M., "Does Quantum Mechanics Accept a Stochastic Support?" Found. Phys. 12, 10, 1982, pp. 1017-1037. 
[14] Barut, A. O., "Schrödinger's Interpretation of $\psi$ as a Continuous Charge Distribution," Ann. der Phys. 7, 4-5, 1988, pp. 31-36.

[15] Barut, A. O., and Bracken, A. J., "Zitterbewegung and the Internal Geometry of the Electron," Phys. Rev. D 23, 10, 1981, pp. 2454-2463.

[16] Vigier, J. P., "De Broglie Waves on Dirac Aether: A Testable Experimental Assumption," Lett. Nuvo Cim. 29, 14, 1980, pp. 467-475; Gueret, Ph., and Vigier, J. P., "De Broglie's Wave Particle Duality in the Stochastic Interpretation of Quantum Mechanics: A Testable Physical Assumption," Found. Phys. 12, 11, 1982, pp. 1057-1083; Cufaro Petroni, C., and Vigier, J. P., "Dirac's Aether in Relativistic Quantum Mechanics," Found. Phys. 13, 2, 1983, pp. 253-286; Vigier, J. P., "Derivation of Inertia Forces from the Einstein-de BroglieBohm (E.d.B.B) Causal Stochastic Interpretation of Quantum Mechanics," Found. Phys. 25, 10, 1995, pp. 1461-1494.

[17] Arecchi, F. T., and Harrison, R. G., Instabilities and Chaos in Quantum Optics, Springer-Verlag, Berlin, 1987.

[18] Reynolds, O., "On the Dynamical Theory of Incompressible Viscous Fluid and the Determination of the Criterion," Phil. Trans. Roy. Soc. A 186, 1, 1895, pp. 23-164.

[19] Taylor, G. I., "Statistical Theory of TurbulenceParts I-IV," Proc. Roy. Soc. A 151, 873, 1935, pp. 421-478.

[20] Kármán, T. von, and Howarth, L., "On the Statistical Theory of Isotropic Turbulence," Proc. Roy. Soc. A 164, 917. 1938, pp. 192-215.

[21] Robertson, H. P., "The Invariant Theory of Isotropic Turbulence," Proc. Camb. Phil. Soc. 36, 1940, pp. 209-223.

[22] Kolmogoroff, A. N., "Local Structure on Turbulence in Incompressible Fluid," C. R. Acad. Sci. U. R. S. S. 30, 1941, pp. 301-305; "Dissipation of Energy in Locally Isotropic Turbulence," C. $R$. Acad. Sci. U. R. S. S. 32, 1942, p. 19-21; “A Refinement of Previous Hypothesis Concerning the Local Structure of Turbulence in a Viscous Incompressible Fluid at High Reynolds Number," J. Fluid Mech. 13, 1962, pp. 82-85.

[23] Obukhov, A. M., "On the Distribution of Energy in the Spectrum of Turbulent Flow," C. R. Acad. Sci. U. R. S. S. 32, 1941, pp. 19-22; "Some Specific Features of Atmospheric Turbulence," J. Fluid Mech. 13, 1962, pp. 77-81.

[24] Chandrasekhar, S., "Stochastic Problems in Physics and Astronomy," Rev. Mod. Phys. 15, 1, 1943, pp. 1-89.

[25] Chandrasekhar, S., Stochastic, Statistical, and Hydrodynamic Problems in Physics and Astronomy, Selected Papers, vol.3, University of Chicago Press, Chicago, 1989, pp. 199-206.

[26] Heisenberg, W., "On the Theory of Statistical and Isotropic Turbulence," Proc. Roy. Soc. A 195,
1948, pp. 402-406; "Zur Statistischen Theorie der Turbulenz,“'Z. Phys. 124, 7-12, 1948, p. 628-657.

[27] Batchelor, G. K., The Theory of Homogeneous Turbulence, Cambridge University Press, Cambridge, 1953.

[28] Landau, L. D., and Lifshitz, E. M., Fluid Dynamics, Pergamon Press, New York, 1959.

[29] Tennekes, H., and Lumley, J. L., A First Course In Turbulence, MIT Press, 1972.

[30] Sohrab, S. H., "Transport Phenomena and Conservation Equations in Multicomponent Chemically-Reactive Ideal Gas Mixtures," Proceeding of the 31st ASME National Heat Transfer Conference, HTD-Vol. 328, 1996, pp. 3760.

[31] Sohrab, S. H., "A Scale Invariant Model of Statistical Mechanics and Modified Forms of the First and the Second Laws of Thermodynamics," Rev. Gén. Therm. 38, 1999, pp. 845-853.

[32] Sohrab, S. H., "Derivation of Invariant Forms of Conservation Equations from the Invariant Boltzmann Equation," In: Theoretical and Experimental Aspects of Fluid Mechanics, S. H. Sohrab, H. C. Catrakis, and F. K. Benra (Eds.), WSEAS Press, 2008, ISBN: 978-960-6766-30-5, pp. 27-35.

[34] Sohrab, S. H., "Universality of a Scale Invariant model of Turbulence and its Quantum Mechanical Foundation," In: Recent Advances in Fluid Mechanics \& Aerodynamics, S. Sohrab, H. Catrakis, and. N. Kobasko (Eds.), WSEAS Press, 2009, ISBN: 978-960-474-106-9, pp. 134-140.

[34] Sohrab, S. H., "Invariant Planck Energy Distribution Law and its Connection to the Maxwell-Boltzmann Distribution Function," WSEAS Transactions on Mathematics 6, 2, 2007, pp. 254-262.

[35] Sohrab, S. H., "Quantum Theory of Fields from Planck to Cosmic Scales," WSEAS Transactions on Mathematics 9, 8, 2010, pp. 734-756.

[36] Enskog, D., "Kinetische Theorie der Vorgange in Massig Verdunnten Gasen," by Almqvist and Wiksells Boktryckeri-A.B., Uppsala, 1917. English translation in Ref. [60], pp. 125-225.

[37] de Groot, R. S., and Mazur, P., Nonequilibrium Thermodynamics, North-Holland, 1962.

[38] Schlichting, H., Boundary-Layer Theory, McGraw Hill, New York, 1968.

[39] Williams, F. A., Combustion Theory, Addison Wesley, New York, 1985.

[40] Hirschfelder, J. O., Curtiss, C. F., and Bird, R. B., Molecular Theory of Gases and Liquids, Wiley, New York, 1954.

[41] Chapman, S., and Cowling, T. G., The Mathematical Theory of Non-uniform Gases, Cambridge University Press, Cambridge, 1953.

[42] Sohrab, S. H., "On a scale invariant model of statistical mechanics, kinetic theory of ideal gas, and Riemann hypothesis," In: Recent Research in Circuits \& Systems, $16^{\text {th }}$ International Conference 
on Systems, E. Balas, M. Koksal, and V Vasek (Eds.), pp: 505-546, WSEAS Press, 2012, ISBN: 978-1-61804-108-1.

[43] van der Waerden, B. L., "Towards Quantum Mechanics," Sources of Quantum Mechanics, B. L. van der Waerden (Ed.), Dover, New York, 1967, pp. 1-59.

[44] Casimir, H. B. G., "On the Attraction between Two Perfectly Conducting Plates," Proc. K. Ned. Akad. Wet. 51, 1948, pp. 793-795.

[45] Planck, M., The Theory of Heat Radiation, Dover, New York, 1991.

[46] Planck, M., "On the Law of the Energy Distribution in the Normal Spectrum," Ann. der Phys. 4, 1901, pp. 553-558.

[47] Jackson, J. D., Classical Electrodynamics, Wiley, New York, 1975.

[48] Newton, I., Optics, Dover, New York, 1952.

[49] Broglie, L., Le Mécanique Ondulatoire du Photon 1, Paris: Herman et Cie, 1940.

[50] Bass, L., and Schrödinger, E., "Must the Photon Mass be Zero?" Proc. Roy. Soc. A 232, 1-6, 1955, pp. 654-661.

[51] Vigier, J. P., "Possible Test of the Reality of Superluminal Phase Waves and Particle Phase Space Motions in the Einstein-de-Broglie-Bohm Causal Stochastic Interpretation of Quantum Mechanics," Found. Phys. 24, 1, 1994, pp. 61-83.

[52] de Beauregard, O. Costa., "The Great Vail, Reality, and Louis de Broglie: Personal Memories," In: Waves and Particles in Light and Matter. A. van der Merwe, and A. Garuccio (Eds.), Plenum Press, New York, 1994, pp. 1-7.

[53] Evans, M. W., "On Longitudinal Free Space-time Electric and Magnetic Fields in the Einstein-de Broglie Theory of Light," In: Waves and Particles in Light and Matter. Alwyn van der Merwe and Augusto Garuccio (Eds.), Plenum Press, New York, 1994, pp. 259-294.

[54] Sohrab, S. H., "The Nature of Mass, Dark Matter, and Dark Energy in Cosmology and the Foundation of Relativistic Thermodynamics," In: New Aspects of Heat Transfer, Thermal Engineering, and Environment, S. H. Sohrab, H. J. Catrakis, N. Kobasko, (Eds.), WSEAS Press, 2008, ISBN: 978-960-6766-97-8, pp. 434-442.

[55] Pauli, W., Pauli Lectures on Physics, Vol.3, MIT Press, 1973, p. 14.

[56] Long, C. A., and Sohrab, S. H., "The Power of Two, Speed of Light, Force and Energy and the Universal Gas Constant," In : Recent Advances on Applied Mathematics, Long, C. A., Sohrab, S. H., Bognar, G., and Perlovsky, L., (Eds.), WSEAS Press, 2008, pp. 434-442

[57] De Pretto, O., “Ipotesi dell'Etere Nella Vita dell'Universo," Reale, Inst. Veneto di Scienze, Lettere en Arti 63, 2, 1904, pp. 439-500.
[58] Poincaré, H., "La Théorie de Lorentz et le Principe de Réaction," Arch. Neerland. 5, 2, 1900, pp.252278; $\quad$ http://www.physicsinsights.org/poincare1900.pdf.

[59] Hasenöhrl, F., "Zur Theorie der Strahlung in bewegten Körpern," Ann. der Physik 15, 1904, pp. 344-370; "Zur Theorie der Strahlung in bewegten Körpern,” Ann. der Phys. 16, 1905, pp. 589-592.

[60] Einstein, A., "Ist die Trägheit eines Körpers von seinem Energieinhalt abhängig?" Ann. der Phys. (Leipzig) 18, 1905, pp. 639-641.

[61] Sommerfeld, H., Die Bedeutung der Röntgenstrahlen für die heutige Physik, Munich, 1925, p. 11. Cited in Weyl, H. Philosophy of Mathematics and Natural Science, Princeton, 1949.

[62] Weyl, H., "Gravitation and Electricity," In: The Principles of Relativity, Dover, New York, 1956, pp. 201-216.

[63] Shannon, C. E., The Mathematical Theory of Communication, Bell System Tech. J. 27, pp. 379423 and 623-656 (1948).

[64] Weaver, W., and Shannon, C. E., The Mathematical Theory of Communication, University of Illinois Press, Chicago, 1963.

[65] Boltzmann, L., "Weitere Studien uber das Warmegleichgewicht unter Gasmoleculen," Sitzungsberichte Akad.Wiss., Vienna, Part II, 66, 1872, pp. 275-370. English Translation in Ref. [67], pp. 88-175.

[66] Boltzmann, L., Lectures on Gas Theory, Dover, New York, 1964.

[67] Brush, G., S., Kinetic Theory, Vol.1-3, Pergamon Press, New York, 1965.

[68] Darrigol, O., "Statistics and Combinatorics in Early Quantum Theory," Historical Studies in the Physical and Biological Sciences, 19, 1, 1988, pp. 17-80; "Statistics and Combinatorics in Early Quantum Theory, II: Early Symptoms of Indistinguishability and Holism," 21, 2, 1991, pp. 237-298.

[69] Sonntag, R. E., Van Wylen, G. E., Fundamentals of Statistical Thermodynamics, Wiley, New York, 1966.

[70] Brillouin, L., Maxwell Demon cannot operate: Information and Entropy I, J. Appl. Phys. 22, 334337 (1951).

[71] Ben-Naim, A., Entropy Demystified, World Scientific, New York, 2008.

[72] Casimir, H. B. G., "On the Attraction between Two Perfectly Conducting Plates,", Proc. K. Ned. Akad. Wet. 51, 793 (1948).

[73] Planck, M., Where Is Science Going, Ox Bow Press, CT, 1981.

[74] Ben-Naim, A., A Farewell to Entropy: Statistical Thermodynamics Based on Information, World Scientific, New York, 2008.

[75] Finn, C. B. P., Thermal Physics, Chapman \&Hall, New York, 1986.

Creative Commons Attribution License 4.0 (Attribution 4.0 International, CC BY 4.0)

This article is published under the terms of the Creative Commons Attribution License 4.0

https://creativecommons.org/licenses/by/4.0/deed.en_US 